\title{
On a pluri-Gaussian model for three-phase microstructures, with applications to 3D image data of gas-diffusion electrodes
}

\author{
Matthias Neumann ${ }^{\mathrm{a}, *}$, Markus Osenberg ${ }^{\mathrm{b}}$, André Hilger ${ }^{\mathrm{c}}$, David Franzen ${ }^{\mathrm{d}}$, Thomas Turek ${ }^{\mathrm{d}}$, \\ Ingo Manke ${ }^{\mathrm{c}}$, Volker Schmidt ${ }^{\mathrm{a}}$ \\ ${ }^{a}$ Institute of Stochastics, Ulm University, Helmholtzstr. 18, D-89069 Ulm, Germany \\ ${ }^{b}$ Department of Materials Science and Technology, TU Berlin, Hardenbergstr. 36, 10623 Berlin, Germany \\ ${ }^{c}$ Institute of Applied Materials, Helmholtz-Zentrum Berlin, Hahn-Meitner-Platz 1, D-14109 Berlin, Germany \\ ${ }^{d}$ Institute of Chemical and Electrochemical Process Engineering, TU Clausthal, Leibnizstr. 17, \\ D-38678 Clausthal-Zellerfeld, Germany
}

\begin{abstract}
A pluri-Gaussian model for three-phase microstructures is presented and relationships between model parameters and microstructure characteristics are discussed. In particular, analytical formulas for two-point coverage probability functions in terms of covariance functions of the underlying Gaussian random fields are considered, which allow for an efficient estimation of model parameters. The model is fitted to tomographic image data obtained by FIB-tomography, which represent porous gas-diffusion electrodes consisting of silver and polytetrafluorethylene. The considered type of electrode is used as oxygen depolarized cathode for the production of chlorine. In order to fit the microstructure model, the covariance functions of the Gaussian random fields are parameterized, which leads to a stochastic microstructure model with five parameters. It is shown that most microstructure characteristics of tomographic image data are well reproduced by the model despite the low number of model parameters. Finally, limitations of the model with respect to the fit of continuous phase size distributions are discussed. Combining stochastic microstructure modeling with numerical simulation of effective macroscopic properties will allow in future work for a model-based investigation of microstructure-property relationships for the considered gas-diffusion electrodes.
\end{abstract}

Keywords: FIB tomography, gas-diffusion electrode, Gaussian random field, image analysis, stochastic microstructure modeling

\section{Introduction}

Macroscopic properties of various functional materials, like e.g. electrode material in fuel cells [1] and batteries [2], are strongly influenced by their underlying microstructure. For such materials, it is thus important to investigate the quantitative relationships between production parameters and microstructure characteristics on the one hand and between microstructure characteristics and effective macroscopic properties, like e.g. effective conductivity or diffusivity, on the other hand. To do so, the generation of virtual but realistic microstructures by means of stochastic modeling has become a powerful tool [3]. Having fitted a parametric stochastic model to tomographic image

${ }^{*}$ Corresponding author. Phone: +49731 50 23617. Email: matthias.neumann@uni-ulm.de. 
data representing microstructures manufactured with different constellations of production parameters, an interpolation of model parameters would allow for a prediction of microstructures which have not been manufactured so far [4]. In this way, the influence of production parameters on the microstructure can be studied. Furthermore, a large amount of various virtual microstructures

$$
\text { can be generated. By a combination with numerical simulation of effective macroscopic properties, }
$$
empirical formulas have been derived, relating effective conductivity with microstructure characteristics [5].

Microstructures consisting of three phases are of high importance because of their frequent occurrence in functional materials, as e.g., in silver-based gas-diffusion electrodes (GDE) for chloralkali electrolysis $[6,7]$ as well as in electrodes of Li-ion batteries [8, 9] and solid oxide fuel cells (SOFC) [10, 11]. Besides stochastic 3D microstructure models based on random geometric graphs [12], collective re-arrangement algorithms [13, 14] and grain growth modeling with random seed points [15], a frequently used model type is the so-called pluri-Gaussian model (PGM) fitted to SOFC microstructures in $[16,17,18]$ and mathematically described in $[19,20]$. The idea of the PGM consists of modeling the three phases by the aid of two independent Gaussian random fields [21]. Each point in the three-dimensional space is assigned to one of the phases according to the values of the Gaussian random fields at this point. In the present paper, we consider a classical generalization of the PGM for three-phase microstructures discussed in [16]. More precisely, we use two correlated Gaussian random fields, see also [20]. We construct them from two independent Gaussian random fields as in [22], where four-phase structures are modeled by excursion sets of random fields. In contrast to the interactive fitting of model parameters considered in [22, Section $4.2]$, we propose a method for parameter estimation by taking advantage of relationships between model parameters and microstructure characteristics.

The practical applicability of our approach is demonstrated at the example of three-phase microstructures occuring in silver-based GDE, which can be well described by the PGM. The model parameters are estimated by means of tomographic image data obtained by FIB-tomography. The considered GDE is used as oxygen depolarized cathode (ODC), a technology for the production of chlorine, which has the advantage to suppress hydrogen evolution and to save up to $30 \%$ of the energy consumption [7, 23, 24] compared to industrial chlor-alkali processes [25, 26]. The electrode consists of silver, polytetrafluorethylene (PTFE) and pores. During the chlor-alkali process electrons are transported through the silver phase, while water and gaseous species, and ions are transported through the pores. Thereby, it is important for both, transport of gas and liquid, that the pore space exhibits hydrophobic as well as hydrophilic regions, which is controlled by the spatial distribution of PTFE being hydrophobic. Chemical reactions at the interface between silver and pores lead to a reduction of oxygen to hydroxyl ions resulting in the suppression of hydrogen evolution. For a detailed description of silver-based GDE, we refer to [7]. Due to the different transport processes taking place in the GDE, the underlying microstructure plays an important role for its performance. Fitting the PGM to the microstructure of silver-based GDE is a first step in order to investigate how the microstructure depends on production parameters and how it influences effective properties of the GDE.

The present paper is organized as follows. To begin with, the stochastic microstructure model considered in this paper is explained in Section 2 and analytical properties of this model are considered, which are used in Section 3 to develop a method for the estimation of model parameters. The model is then fitted to tomographic image data obtained by FIB-tomography, which describes the 
of the considered GDE are described in Sections 4.1 and 4.2, respectively. Finally, in Section 4.3, model fitting is discussed and a validation based on microstructure characteristics which have not been used for model fitting is provided. Section 5 concludes the paper.

\section{Model description}

5 fields $X=\left\{X(t): t \in \mathbb{R}^{3}\right\}$ and $Y=\left\{Y(t): t \in \mathbb{R}^{3}\right\}$, with covariance functions $\rho_{X}$ and $\rho_{Y}$. Moreover, $\mathbb{E} X(t)=\mathbb{E} Y(t)=0$ and $\operatorname{Var} X(t)=\operatorname{Var} Y(t)=1$ for all $t \in \mathbb{R}^{3}$. This means that the random variables $X(t)$ and $Y(t)$ follow the law of the standard normal distribution for each $t \in \mathbb{R}^{3}$. For an introduction to Gaussian random fields and their applications in microstructure modeling 10 and geostatistics, we refer to [19] and [27].

We consider a third random field $Z=\left\{Z(t): t \in \mathbb{R}^{3}\right\}$ given by

$$
Z(t)=\sqrt{m} X(t)+\sqrt{1-m} Y(t)
$$

for each $t \in \mathbb{R}^{3}$, where $m \in[0,1]$ is a certain weight parameter. By the aid of the random fields $X$ and $Z$ and two further threshold parameters $\lambda_{X}, \lambda_{Z} \in \mathbb{R}$ we define three random sets $\Xi_{1}, \Xi_{2}$ and $\Xi_{3}$ by $\Xi_{1}=\left\{t \in \mathbb{R}^{3}: X(t) \geq \lambda_{X}\right\}, \Xi_{2}=\left\{t \in \mathbb{R}^{3}: Z(t) \geq \lambda_{Z}\right\} \cap \Xi_{1}^{c}$ and $\Xi_{3}=\left(\Xi_{1} \cup \Xi_{2}\right)^{c}$, which form 3D microstructure in silver-based GDE by such random sets, where $\Xi_{1}$ denotes the silver phase, $\Xi_{2}$ the PTFE and $\Xi_{3}$ denotes the pore space. Note that for $m=0$, we are in the situation of the PGM considered in [16]. Since $X$ and $Y$ are motion invariant, the random sets $\Xi_{1}, \Xi_{2}$ and $\Xi_{3}$ are motion invariant as well. For each $i \in\{1,2,3\}$, we denote the volume fraction of the $i$-th phase by $\varepsilon_{i}$, i.e. $\varepsilon_{i}=\mathbb{P}\left(o \in \Xi_{i}\right)$. Here $o$ denotes the origin in $\mathbb{R}^{3}$. Moreover, for each pair $i, j \in\{1,2,3\}$ we consider the two-point coverage probability function by $C_{i j}$, i.e. $C_{i j}:[0, \infty) \longrightarrow[0,1]$ with $C_{i j}(h)=\mathbb{P}\left(o \in \Xi_{i}, u \in \Xi_{j}\right)$ for each $h \in[0, \infty)$, where $u \in \mathbb{R}^{3}$ is an arbitrary vector with distance $h$ from the origin. Note that the values of $C_{i j}(h)$ do not depend on the particular choice of $u$ due to the motion invariance of $\Xi_{1}, \Xi_{2}$ and $\Xi_{3}$. Two-point coverage probability functions are an important tool to measure the correlation within and between random sets [27].

In the following we consider some analytical properties of our model. For this purpose, we follow the notation in [28] and let $\varphi(t)$ denote the value of the probability density function of the standard normal distribution at $t$. By $\Phi(t)$, we denote the value of the distribution function of the standard normal distribution at $t$. Moreover, the value of the probability density function of a two-dimensional Gaussian random vector, both entries of which have mean value 0 , variance 1 and correlation coefficient $-1<\gamma<1$, evaluated at $(s, t)$ is denoted by $\varphi(s, t, \gamma)$. It is well known that

$$
\varepsilon_{1}=1-\Phi\left(\lambda_{X}\right)
$$

see [27]. For the volume fraction $\varepsilon_{2}$ of $\Xi_{2}$, we have

$$
\begin{aligned}
\varepsilon_{2} & =\mathbb{P}\left(\sqrt{m} X(o)+\sqrt{1-m} Y(o) \geq \lambda_{Z}, X(o)<\lambda_{X}\right) \\
& =\int_{-\infty}^{\lambda_{X}} \varphi(t)\left(1-\Phi\left(\frac{\lambda_{Z}-t \sqrt{m}}{\sqrt{1-m}}\right)\right) \mathrm{d} t .
\end{aligned}
$$

Thus, using this formula, we can estimate $\lambda_{X}$ and $\lambda_{Z}$ indirectly by estimating the volume fractions $\varepsilon_{1}$ and $\varepsilon_{2}$. In order to estimate $\rho_{X}, \rho_{Y}$ and $m$ the following relationships between covariance 
functions and two-point coverage probability functions are helpful. The following relationship between $\rho_{X}$ and $C_{11}$ is well known $[19,27]$. Namely, it is

$$
C_{11}(h)=\varepsilon_{1}^{2}+\int_{0}^{\rho_{X}(h)} \varphi\left(\lambda_{X}, \lambda_{X}, \gamma\right) \mathrm{d} \gamma
$$

for each $h \geq 0$. Furthermore, $C_{22}$ and $C_{12}$ can be expressed by $\lambda_{X}, \lambda_{Z}, \rho_{X}$ and $\rho_{Y}$. Let $u \in \mathbb{R}^{3}$ be an arbitrary vector of length $h$. Then, for $C_{22}$, we have

$$
\begin{aligned}
C_{22}(h)= & \mathbb{P}\left(\sqrt{m} X(o)+\sqrt{1-m} Y(o) \geq \lambda_{Z}, X(o)<\lambda_{X},\right. \\
& \left.\sqrt{m} X(u)+\sqrt{1-m} Y(u) \geq \lambda_{Z}, X(u)<\lambda_{X}\right) \\
= & \int_{-\infty}^{\lambda_{X}} \int_{-\infty}^{\lambda_{X}} \varphi\left(s, t, \rho_{X}(h)\right) \int_{\frac{\lambda_{Z}-s \sqrt{m}}{\sqrt{1-m}}}^{\infty} \int_{\frac{\lambda_{Z}-t \sqrt{m}}{\sqrt{1-m}}}^{\infty} \varphi\left(\widetilde{s}, \widetilde{t}, \rho_{Y}(h)\right) \mathrm{d} \widetilde{s} \mathrm{~d} \widetilde{t} \mathrm{~d} s \mathrm{~d} t .
\end{aligned}
$$

For $C_{12}$, we obtain that

$$
\begin{aligned}
C_{12}(h) & =\mathbb{P}\left(\sqrt{m} X(o)+\sqrt{1-m} Y(o) \geq \lambda_{Z}, X(o)<\lambda_{X}, X(u) \geq \lambda_{X}\right) \\
& =\int_{-\infty}^{\lambda_{X}} \int_{\lambda_{X}}^{\infty} \varphi\left(s, t, \rho_{X}(h)\right)\left(1-\Phi\left(\frac{\lambda_{Z}-t \sqrt{m}}{\sqrt{1-m}}\right)\right) \mathrm{d} s \mathrm{~d} t .
\end{aligned}
$$

In Section 3 we show how Equations (2) - (5) can be used to estimate the model parameters from image data. For this purpose, i.e. for fitting the model to tomographic image data, we appropriately parameterize the covariance functions $\rho_{X}$ and $\rho_{Y}$ see Section 4.3 below.

\section{Estimation of model parameters}

In this section, a method is described in order to estimate the model parameters $\lambda_{X}$ and $\lambda_{Z}$ as well as the covariance functions $\rho_{X}$ and $\rho_{Y}$ based on one realization of the generalized PGM. To begin with, estimators $\widehat{\varepsilon}_{1}, \widehat{\varepsilon}_{2}, \widehat{C}_{11}$ and $\widehat{C}_{22}$ for $\varepsilon_{1}, \varepsilon_{2}, C_{11}$ and $C_{22}$ are computed from image data. Then, we solve Equations (2) - (5) numerically for the model parameters sequentially as sketched in Figure 1 for some fixed $m \in[0,1]$. In the next step, this procedure is repeated for several values of $m$. Finally $\widehat{m}$ is chosen as the value of $m$ for which the mean squared difference between $\widehat{C}_{12}$ estimated from image data and $C_{12}$ numerically computed with the considered model parameters using Equation (6) is minimized.

For estimating the volume fractions $\varepsilon_{1}$ and $\varepsilon_{2}$, we use the usual point-count method [27], i.e. counting the voxels of the considered phase, and divide the result by the number of all voxels in the sampling window. To estimate the two-point coverage probability functions $C_{11}$ and $C_{22}$ from image data, we use the algorithm from [29] which is based on the fast Fourier transformation.

Then, $\lambda_{X}$ can be estimated via

$$
\widehat{\lambda}_{X}=\Phi^{-1}\left(1-\widehat{\varepsilon}_{1}\right) .
$$

In the next step, $\lambda_{Z}$ and $\rho_{X}$ are estimated by plugging the estimators $\widehat{\varepsilon}_{1}$ and $\widehat{C}_{11}(h)$ into Equations (3) and (4) and solving for $\lambda_{Z}$ and $\rho_{X}(h)$ for each $h>0$. Note that the right-hand sides of Equations (3) and (4) are monotonous in $\lambda_{Z}$ and $\rho_{X}(h)$ for each $h>0$, respectively. Thus, both

equations can be numerically solved using the method of bisection. For numerical computation of 


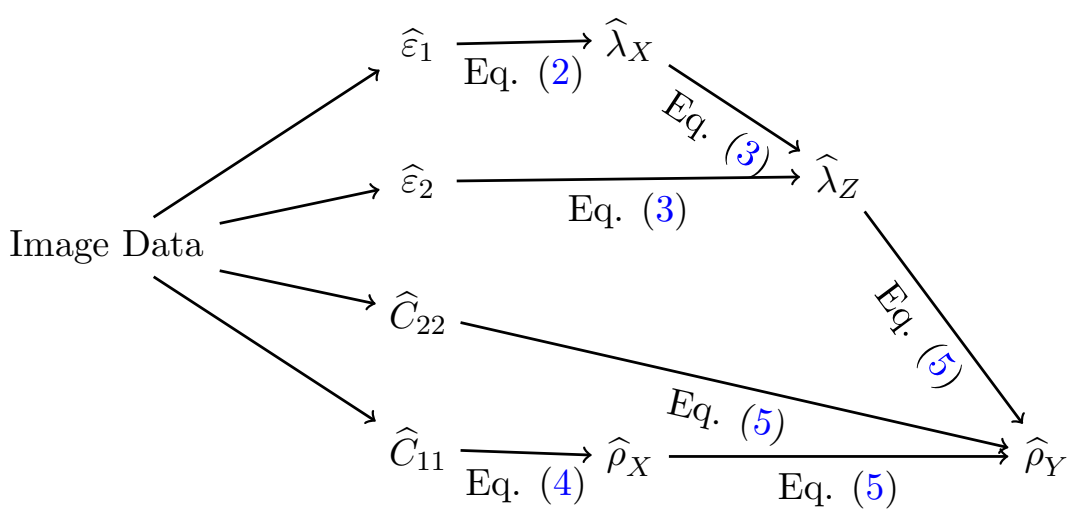

Figure 1: For fixed $m \in[0,1]$, the values of $\lambda_{X}, \lambda_{Z}$ as well as $\rho_{X}$ and $\rho_{Y}$ are estimated sequentially by the aid of Equations (2) - (5). Therefore, $\varepsilon_{1}, \varepsilon_{2}, C_{11}$ and $C_{22}$ are estimated from image data.

the one-dimensional integrals in Equation (3) and (4), we use the adaptive quadrature described in [30]. Plugging the estimators $\widehat{C}_{11}, \widehat{\lambda}_{X}, \widehat{\lambda}_{Z}$ and $\widehat{\rho}_{X}(h)$ into Equation (5) and solving for $\rho_{Y}(h)$, we obtain an estimator $\widehat{\rho}_{Y}(h)$ for $\rho_{Y}(h)$ for each $h>0$. Since the right-hand side of Equation (5) is monotonously increasing in $\rho_{Y}(h)$ the method of bisection can also be used here. For numerical 5 computation of the two-dimensional integral in (5), the method described in [31] is used. To compute the distribution function of the bivariate normal distribution of $(Y(o), Y(u))$, appearing in Equation (5), we use the MATLAB [32] implementation of methods developed in [33] and [34].

This approach allows for a fast estimation of the model parameters based on image data, since it does not involve any simulation of the complete 3D structure. The estimation is only based on volume fractions and two-point coverage probability functions, which can be easily estimated using tomographic image data. Note that even 2D images are sufficient to estimate these characteristics, which is a big advantage. However, taking the full 3D information into account leads to a larger data basis for the estimation and thus reduces the variances of the estimators.

\section{Application to tomographic image data}

We show that the model presented in Section 2 is appropriate to describe the microstructure of porous silver-based GDE treated with PTFE. After a description of the material and 3D image acquisition in Sections 4.1 and 4.2, the model parameters are estimated using a suitable parametric model for the covariance functions $\rho_{X}$ and $\rho_{Y}$, see Section 4.3.

\subsection{Description of material}

The considered cathodes are produced by a wet preparation process. For a detailed description, the reader is referred to [7]. A suspension with the silver catalyst, PTFE as an organic binder and a methyl cellulose solution as a pore building agent and thickener is applied on a conductive supporting material. The $1 \mathrm{wt} \%$ methyl cellulose solution is prepared by mixing hydroxyethylmethyl cellulose (WALOCEL ${ }^{\mathrm{TM}}$ MKX 70000 PP 01) with demineralized water and stirring the solution for several hours with an Ultra Turrax at $1650 \mathrm{rpm}$. After complete dissolving the solution remains stable and can be used for multiple production batches. The catalytic silver particles (SF9ED, Ferro $\mathrm{GmbH}$ ) were dispersed in the methyl cellulose solution under the addition of water. For a 
homogenous distribution of the particles the suspension is stirred three times for five minutes with an Ultra Turrax at $17500 \mathrm{rpm}$. In between the mixing intervals the dispersion rests to cool down. An aqueous PTFE suspension with 59 wt\% PTFE (PTFE Dispersion TF 5060GZ, 3M ${ }^{\mathrm{TM}}$ Dyneon $^{\mathrm{TM}}$ ) was added under stirring at lower rotational speed. To avoid agglomeration the suspension is stirred

5 permanently.

The suspension is applied by hand spraying on the conductive supporting material, a nickel mesh $(106 \mu \mathrm{m} \times 118 \mu \mathrm{m}$ mesh size, $63 \mu \mathrm{m}$ thickness, Haver \& Boecker OHG), using a spraying piston with a $0.6 \mathrm{~mm}$ pin hole. The nickel mesh is fixed on a heating plate which allows simultaneous drying while spraying. Multiple layers are applied, each new layer after sufficient drying of the previous one. After every tenth layer the electrode is rotated to avoid sticking on the heating plate and to generate a homogeneous surface. This procedure is repeated until a silver loading of $150 \mathrm{mg} / \mathrm{cm}^{2}$ is reached. To improve the physical properties the electrode is pressed with a pressing load of $15 \mathrm{MPa}$ at $130^{\circ} \mathrm{C}$ for five minutes. After pressing the electrode is heat treated at $330^{\circ} \mathrm{C}$ for 15 minutes to burn out the methyl cellulose and improve the mechanical stability through PTFE sintering. The heating rate of the sintering process is $3^{\circ} \mathrm{C} / \mathrm{min}$ while after several heating steps the temperature is kept constant for 15 minutes. After a cooldown step the electrode thickness and catalyst load is verified.

\subsection{D FIB-SEM imaging}

To investigate the 3D microstructure of the electrodes described in Section 4.1, 3D FIBtomography is performed, which is challenging for porous materials. Filling the pores with material can change the sample structure or cause contrast problems. Especially regarding the contrast of the PTFE and the filling material. For tomographies of large volumes (more than $1000^{3}$ voxels), long exposures, which would lead to a better signal to noise ratio and thus to a better material contrast, are not practical. The main problem here is the long time stability of the FIB-SEM system. For the silver-based GDE analyzed in the present paper, pore filling was tested with the epoxy-resin based EpoThin ${ }^{\mathrm{TM}_{2}}$ and Technivit 5071 and the silicon based Wacker Elastosil RT 601 resulting in no satisfactory material contrasts.

In the present work, we use an alternative technique without pore filling for FIB-tomography. A FIB-tomography on porous materials requires much more post processing than a FIB-tomography on embedded materials. Reconstruction has to be done very carefully removing shine-through artifacts and classifying only the cutting surface of the sample.

A $3 \times 1 \mathrm{~mm}^{2}$ segment is cut out of the center of the electrode. For stabilization, the segment is then embedded into EpoThin ${ }^{\mathrm{TM}_{2}}$ mixing the epoxy resin and the epoxy hardener with a 20:9 ratio. To prevent the adhesive from entering the pore system of the sample, the mixture is applied after 3 hours in a partly cured state. After full adhesive curing, an edge of the sample is mechanically grinded and polished perpendicularly to the surface normal of the sample. The applied polishing procedure leads to a cross-section of the sample making a FIB-tomography of a representative part in the center of the sample possible. For final mechanical polishing, a colloidal silica suspension (OP-U NonDry) is used. Note that polishing porous silver results in a smearing of the silver material into the pores which makes a non-mechanical polishing step necessary. Further polishing the sample with a Bal-Tec RES 101 argon ion mill with an acceleration voltage of $3 \mathrm{KeV}$ and a current of 1.5 $\mathrm{mA}$ for 2 hours at a glancing angle of 5 degrees removes the upper most layer of smeared silver and uncovers the final cross-section of the electrode for FIB-tomography.

FIB-tomography is performed using the Zeiss Crossbeam 340 microscope. Gallium ion milling parameters are set to an acceleration voltage of $30 \mathrm{KeV}$ and a current of $7 \mathrm{nA} .1300$ slices with 
a track spacing of $30 \mathrm{~nm}$ are scanned over a time period of 6 hours. The voxel size is $30 \mathrm{~nm}$ in all directions. An $1103 \times 979 \times 690$ voxel volume has been reconstructed successfully. For further analysis a good correction of the intensity gradients is important. Due to the U-shape of the premilled volume during preprocessing, the intensity gradient of the tomographed slices only 5 changes vertically. A maximum projection of the resliced volume (from left to right) leads to an excellent volume-flatfield and thus to a precise normalization of the silver phase. The pore space is reconstructed using a machine learning approach based on the ideas proposed in [35, 36] utilizing SE2-detector shadow effects in milling direction. Hence the drift corrected image set is denoised using the non-local means filter [37] and the discrete derivatives of first and second order in milling direction have been analyzed and segmented with the ImageJ software package Fiji [38]. After classification of the pore space, the PTFE- and the silver-phase have been separated by applying an Otsu-threshold [39].
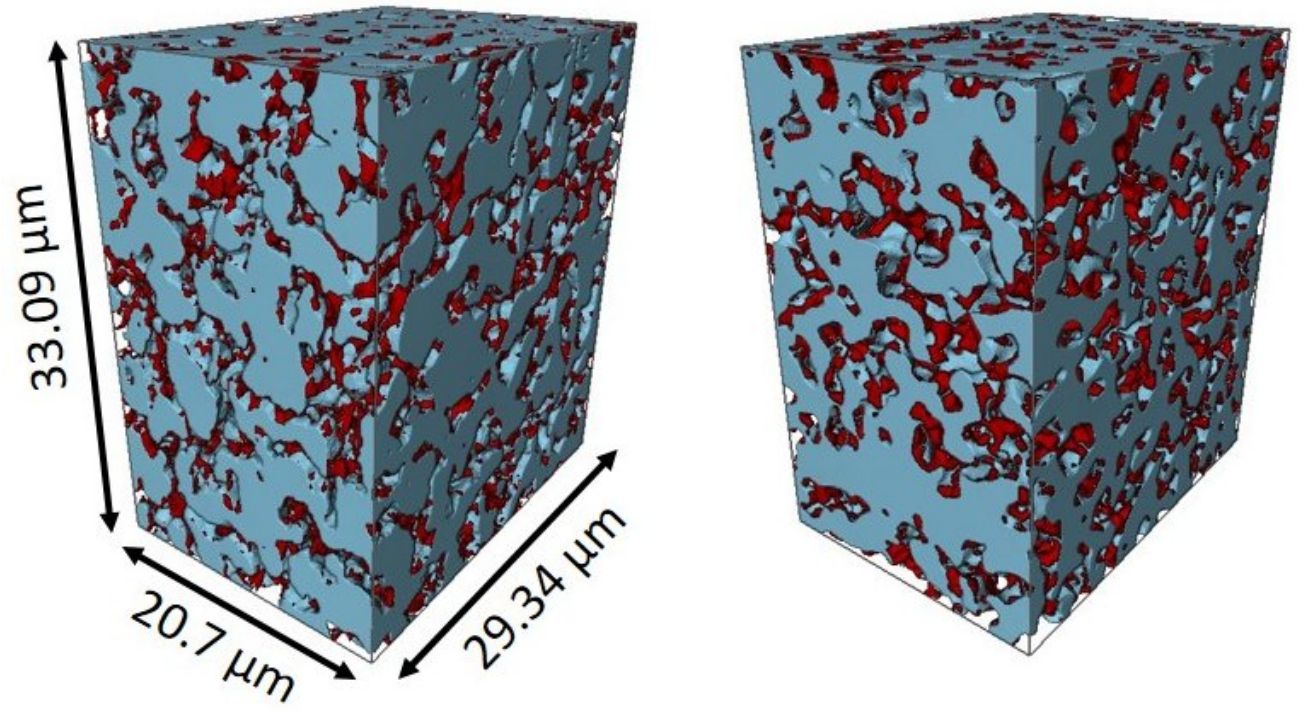

Figure 2: 3D visualization of tomographic image data (left) and one model realization (right). The silver phase and PTFE are represented in blue and red, respectively.

\subsection{Model fitting and validation}

In this section, the results of model fitting to tomographic image data are described. Recall from Section 2 that the silver phase, PTFE and the pore space are modeled by the random sets $\Xi_{1}, \Xi_{2}$ and $\Xi_{3}$, respectively, which are constructed by Gaussian random fields. This approach is appropriate to model microstructures of GDE, manufactured as described in Section 4.1, for two reasons. First, note that the pluri-Gaussian model is hierarchical in the sense that we begin with determining $\Xi_{1}$ before its complement is subdivided into $\Xi_{2}$ and $\Xi_{3}$. This subdivision corresponds to the sintering in the last step of the manufacturing process of the considered GDE, when the silver phase remains unchanged and the PTFE accumulates around the silver phase. Second, the model has the advantage that we can control how strong the PTFE accumulates around the silver phase by adjusting the model parameter $m$. Mathematically speaking, the correlation between $\Xi_{1}$ and $\Xi_{2}$, quantified by the two-point coverage probability function $C_{12}$, can be controlled by a suitable choice of $m$. 
For parameter estimation, we only consider every second voxel in order to reduce the computational effort. Finally, the goodness of model fit is validated by computing structural characteristics for both, 10 model realizations and tomographic image data. For this purpose, discretized model realizations are generated, which can be performed by the simulation of the two independent Gaussian random fields $X$ and $Y$ as described in [16].

Table 1: Model parameters estimated from tomographic 3D image data.

\begin{tabular}{ccccc}
$\lambda_{X}$ & $\lambda_{Z}$ & $\alpha_{X}$ & $\alpha_{Y}$ & $m$ \\
\hline-0.21 & -0.20 & $0.99 \mu \mathrm{m}^{-1}$ & $2.02 \mu \mathrm{m}^{-1}$ & 0.86
\end{tabular}

In order to fit the model to the tomographic image data representing the microstructure of cathodes, described in Section 4.1, we use a parametric model for the covariance functions $\rho_{X}, \rho_{Y}$ of $X$ and $Y$. In particular, after having estimated $C_{11}$ and $C_{22}$ non-parametrically from image data we compute non-parametric estimates of $\rho_{X}$ and $\rho_{Y}$, as described in Section 3, denoted by $\widehat{\rho}_{X}$ and $\widehat{\rho}_{Y}$, respectively. It turned out that a good fit is obtained for $\widehat{C}_{11}$ and $\widehat{C}_{22}$ when assuming $\rho_{X}$ and $\rho_{Y}$ to be Gaussian covariance functions, i.e. $\rho_{X}(h)=\exp \left(-\left(\alpha_{X} h\right)^{2}\right)$ and $\rho_{Y}(h)=\exp \left(-\left(\alpha_{Y} h\right)^{2}\right)$ for each $h>0$, where $\alpha_{X}, \alpha_{Y}>0$. This means that, in the following, we consider a parametric stochastic model with the five parameters $\lambda_{X}, \lambda_{Z}, \alpha_{X}, \alpha_{Y}$ and $m$ for three-phase microstructures. Using the approach presented in Section 3, we then estimate these model parameters. The obtained values are listed in Table 1 and a realization drawn from the model with the fitted parameters is visualized in Figure 2.
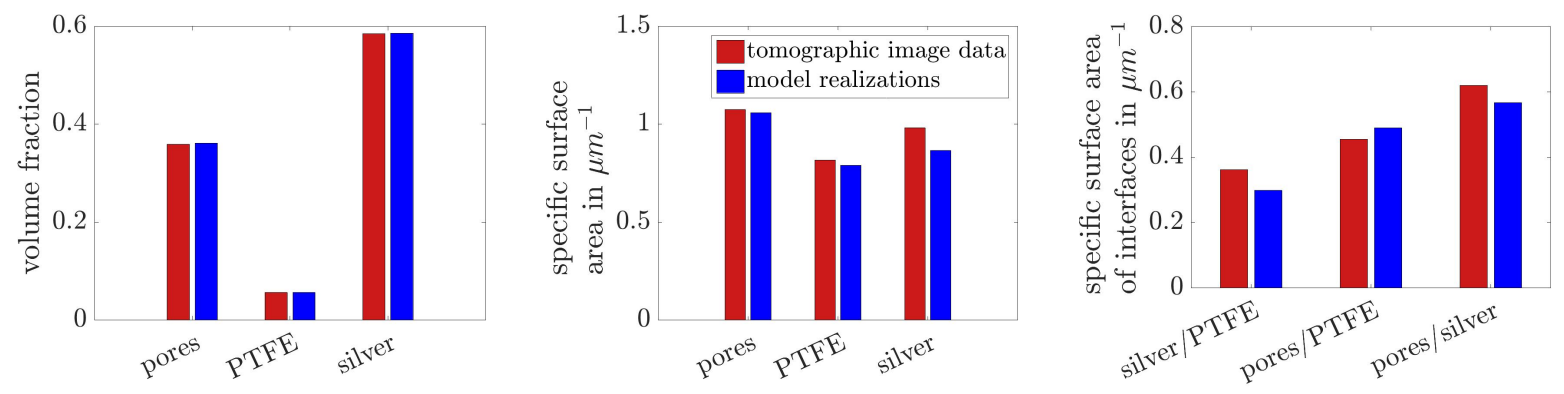

Figure 3: Comparison of volume fractions (left), specific surface areas (center) and specific surface areas of pairwise interfaces (right) for tomographic (red) and simulated (blue) image data. For the simulated, we consider the average of 10 model realizations.

To begin with, we consider volume fractions, specific surface areas and the specific surface areas of pairwise interfaces. Note that the specific surface area of each phase is defined as its expected surface area per unit volume. Analogously, we define the specific surface area of pairwise interfaces between two phases. For the estimation of surface areas from image data, we use the algorithm described in [29]. Furthermore, an analytical expression can be derived for the specific surface area of the silver phase denoted by $S_{\Xi_{1}}$. Indeed, using Equation (6.164) of [27] and Remark 7 of [28], we get that

$$
S_{\Xi_{1}}=a_{X} \frac{2 \sqrt{2}}{\pi} e^{-\lambda_{X}^{2} / 2} .
$$

This means that for a fixed volume fraction of the silver phase, its specific surface area depends linearly on the model parameter $a_{X}$. Plugging the estimated model parameters given in Table 1 into 

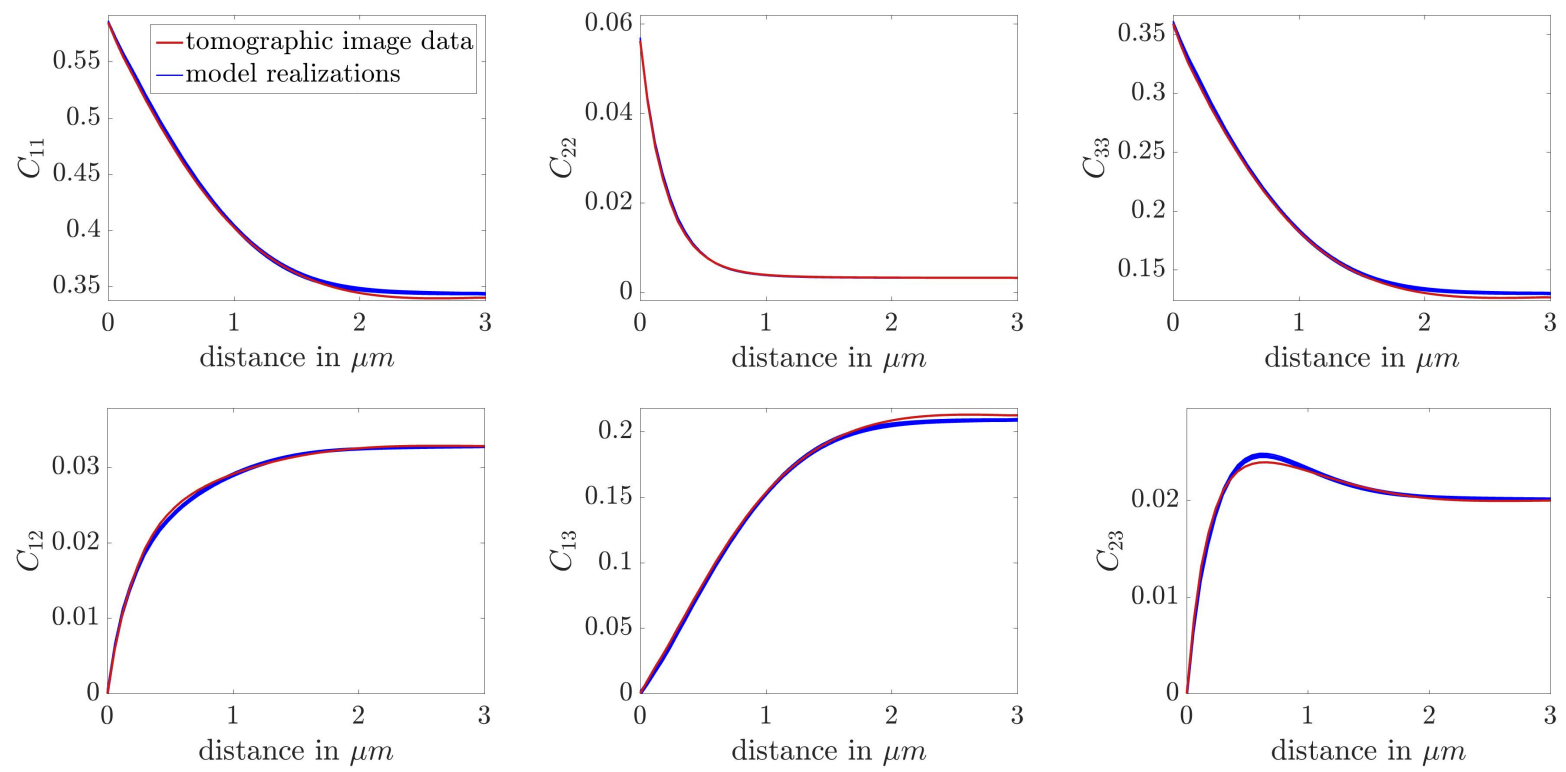

Figure 4: Comparison of tomographic and simulated image data with respect to estimated two-point coverage probability functions $C_{11}, C_{22}, C_{33}$ (top row, from left to right) and $C_{12}, C_{13}, C_{23}$ (bottom row, from left to right). The functions corresponding to tomographic image data and to all 10 model realizations are drawn in red and blue, respectively. Recall that index 1 indicates the silver phase, index 2 the PTFE, and index 3 the pore space.

Equation (8) leads to $S_{\Xi_{1}}=0.872 \mu \mathrm{m}^{-1}$, which is in good accordance with the corresponding value of $0.866 \mu^{-1}$ estimated from discretized model realizations. A comparison of volume fractions, specific surface areas and specific surface areas of pairwise interfaces is given in Figure 3. Here one can observe that the volume fractions, which have been used for model fitting, are nearly identical with those of simulated images drawn from the fitted model. In general, the specific surface areas are also well reproduced by the model. Only the specific surface area of the silver phase is slightly underestimated by the model. Thus the specific surface areas of pairwise interfaces between silver and PTFE as well as between silver and pores are also slightly underestimated.
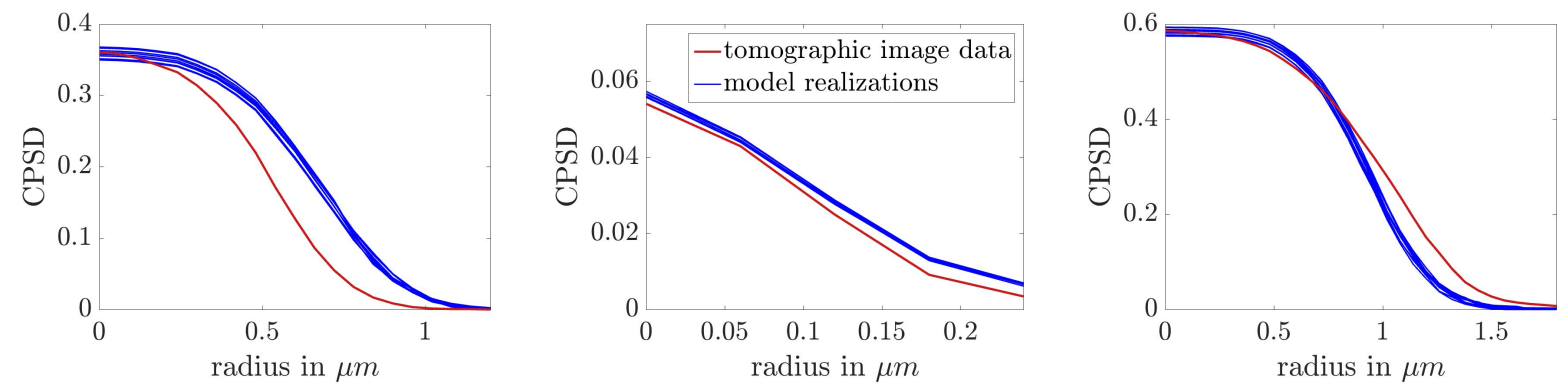

Figure 5: Comparison of continuous phase size distributions of pore space (left), PTFE (center) and the silver phase (right) for tomographic image data (red) and simulated data (blue). For the model, we consider the continuous phase size distributions of all 10 realizations.

Figure 4 shows that the model is able to nicely mimic the considered tomographic image data with respect to two-point coverage probability functions. While the estimators of $C_{11}, C_{22}$ and 
$C_{12}$ computed from tomographic image data are used for model fitting, the good accordance between model realizations and tomographic image data regarding $C_{33}, C_{13}$ and $C_{23}$ can be seen as a validation of the model. Moreover, the estimators of two-point coverage probability functions are nearly identical for the different model realizations, which leads top the conclusion that the chosen sampling window is representative for this type of microstructures.

On the other hand, there are microstructure characteristics which match less perfectly than those considered in Figures 3 and 4. For example, as a further microstructure characteristic, we estimated the continuous phase size distribution (CPSD) [40]. For each $i \in\{1,2,3\}$, the value $P(r)$ of the CPSD of $\Xi_{i}$ at $r>0$ is defined as the volume fraction of the morphological opening [41] of $\Xi_{i}$ with radius $r$, i.e., the volume fraction of the subset of $\Xi_{i}$ that can be represented by spheres with radius $r$ being completely contained in $\Xi_{i}$. Formally, the CPSD of $\Xi_{i}$ is defined as the function $P:[0, \infty) \rightarrow[0,1]$ with

$$
P(r)=\mathbb{P}\left(o \in\left(\Xi_{i} \ominus B(o, r)\right) \oplus B(o, r)\right),
$$

for each $r \geq 0$, where $B(o, r)$ denotes the ball with radius $r$ centered at the origin. Moreover, $\oplus$ and $\ominus$ denote the Minkowski-addition and the Minkowski-subtraction of sets, respectively [27]. Note that the continuous phase size distribution of $\Xi_{i}$ coincides with the size distribution of $\Xi_{i}$ with respect to the spherical granulometry of mathematical morphology [42, Section 7.3].

The plots presented in Figure 5 show that the continuous phase size distribution of the pore space estimated from model realizations differs significantly from the one estimated from tomographic image data. More precise, the model overestimates the occurrence of larger pores. The results shown in Figure 5 also indicate that the fraction of the silver phase that can be covered by spheres of radii larger than $1 \mu \mathrm{m}$ is smaller in the model compared to tomographic image data. However, the continuous phase size distribution of PTFE is well reflected in the model.

Table 2: Transport-relevant microstructure characteristics $\tau, r_{\min }, r_{\max }$ and $\beta$ estimated for tomographic image data and compared to the mean value and standard deviations of these characteristics estimated for 10 model realizations.

\begin{tabular}{lcccc} 
& $\tau$ & $r_{\min }[\mu \mathrm{m}]$ & $r_{\max }[\mu \mathrm{m}]$ & $\beta$ \\
\hline Pore space (tomographic image data) & 1.09 & 0.42 & 0.52 & 0.65 \\
\hline Pore space (model realizations) & $1.12 \pm 0.00$ & $0.42 \pm 0.01$ & $0.62 \pm 0.00$ & $0.47 \pm 0.01$ \\
\hline Silver phase (tomographic image data) & 1.07 & 0.73 & 0.99 & 0.53 \\
\hline Silver phase (model realizations) & $1.06 \pm 0.00$ & $0.72 \pm 0.02$ & $0.89 \pm 0.01$ & $0.065 \pm 0.02$
\end{tabular}

Since transport of gas and liquid through the pores as well as charge transport through the silver phase is important for the functionality of the considered GDE, we still compare the transportrelevant microstructure characteristics mean geodesic tortuosity $\tau$ and constrictivity $\beta$ of the pore space and the silver phase for tomographic image data and model realizations. The mean geodesic tortuosity is defined as the expected length of shortest paths trough from one side of the material to the opposite one divided by the thickness of the material. Note that $\tau$ does only depend on the geometry of the microstructure in contrast to definitions of effective tortuosities considered in the literature. For an overview on different tortuosity concepts, we refer to [43]. The constrictivity, which was introduced in [44] for complex microstructures, is a descriptor of the strength of bottleneck effects within a microstructure. It is defined by $\beta=r_{\min }^{2} / r_{\max }^{2}$, where $r_{\max }$ is the maximum radius such that the value of the continuous pore size distribution is still larger than half of the volume fraction and $r_{\min }$ can be considered as the radius of the characteristic bottleneck. For a formal 
definition of $\tau, r_{\min }, r_{\max }$ and $\beta$ and the corresponding estimators in the framework of stationary random closed sets, we refer to [45]. Note that in [5, 46, 47] it was shown that these microstructure characteristics have a strong impact on effective conductivity and permeability of porous materials. The values of $\tau, r_{\min }, r_{\max }$ and $\beta$ computed for for the tomographic image data considered in the model realizations are given in Table 2 . One can observe that the standard deviations of the characteristics estimated from the 10 different model realizations are negligibly small, which indicates that the sampling window is also large enough with respect to $\tau$ and $\beta$. Moreover, the model shows a good fit with respect to $\tau$ and $r_{\min }$. In particular the values of $r_{\min }$ are nearly identical for model realizations and tomographic image data. Note that such a good fit of $r_{\min }$ was not possible to obtain when fitting the model with $m=0$ to tomographic image data representing the microstructure of SOFC-anodes in [18]. However, in accordance with the results presented in Figure $5, r_{\max }$ is overestimated by the model for the pore space and underestimated for the silver phase. This results then in slightly different values for $\beta$, which means that the strength of bottleneck effects is not yet completely captured by the generalized model considered in the present paper.

In summary, evaluating the goodness of fit of the PGM described in Section 2 to tomographic image data of silver-based GDE, it can be said that the model with only 5 parameters is able to well reproduce basic microstructure characteristics like volume fractions of phases, two-point coverage probability functions as well as specific surface areas. Taking more sophisticated microstructure characteristics into account we observe on the one hand that slight discrepancies between the continuous pore size distributions of model realizations and tomographic image data occur which result in different values of $r_{\max }$ and $\beta$, while on the other hand, a good fit with respect to the radius of the characteristic bottleneck $r_{\min }$ and the mean geodesic tortuosity $\tau$ is obtained.

\section{Conclusions}

A stochastic 3D microstructure model based on excursion sets of Gaussian random fields is presented to model three-phase microstructures. The excursion sets are constructed by two correlated Gaussian random fields, the correlation of which is controlled by a certain weight parameter. Analytical relationships between model parameters and both, volume fractions as well as two-point coverage probability functions of the three-phasesare considered, which allow for an efficient estimation of model parameters.

The model is fitted to image data of silver-based GDE used as ODC to produce chlorine, where the image acquisition is performed by 3D FIB-tomography. For this purpose, we parameterize the covariance functions of the Gaussian random fields ending up in a 3D microstructure model for threephase materials which possesses five parameters. Despite the low model complexity, microstructure characteristics such as the volume fractions of phases, their specific surface areas as well as twopoint coverage probability functions are well reflected by the model. Furthermore, we consider two further transport-relevant microstructure characteristics, namely mean geodesic tortuosity and constrictivity. Mean geodesic tortuosity is fitted well by the model. Although the radii of the characteristic bottlenecks of the pore space and the silver phase are nearly perfectly reproduced by the model, slightly different values of constrictivities are observed for model realizations and tomographic image data. The reason for that are some discrepancies in the continuous phase size distributions.

In future work the model can be used in order to investigate the microstructure influence on effective macroscopic properties of the GDE, e.g. regarding effective conductivity of the silver phase 
and permeability of the pore space, which are important for the overall efficiency of the electrode and thus for the chlorine production. However, before doing so, the stochastic microstructure model considered in the present paper still has to be validated in terms of effective macroscopic properties, which can be achieved by computing the latter ones by means of numerical simulations. If it is necessary to have a better fit of constrictivity in order to adequately reproduce effective macroscopic properties, we can proceed as in [18], where an additional fine-tuning of model parameters has been performed in order to simultaneously fit permeability and effective conductivities of two phases within three-phase microstructures. In this way the present paper can be considered as a first step towards a model-based investigation of microstructure-property relationships for an optimization of the microstructure of silver-based GDE.

\section{Acknowledgements}

The work of M.O., A.H., D.F., T.T., and I.M. was funded by the Deutsche Forschungsgemeinschaft in the framework of the Forschergruppe 'Multi-scale analysis of complex three-phase systems: oxygen reduction on gas-diffusion electrodes in aqueous electrolyte' (FOR 2397).

\section{Data availability}

The raw/processed data required to reproduce these findings cannot be shared at this time as the data also forms part of an ongoing study.

\section{Author contributions}

Research regarding stochastic 3D modeling was carried out by M.N. and V.S. 3D imaging of materials, which have been provided by D.F. and T.T., was performed by M.O., A.H. and I.M.

\section{References}

[1] T. Suzuki, Z. Hasan, Y. Funahashi, T. Yamaguchi, Y. Fujishiro, and M. Awano. Impact of anode microstructure on solid oxide fuel cells. Science, 325(5942):852-855, 2009.

[2] B. Tjaden, D. J. L. Brett, and P. R. Shearing. Tortuosity in electrochemical devices: a review of calculation approaches. International Materials Reviews, 63(2):47-67, 2018.

[3] S. Torquato. Random Heterogeneous Materials: Microstructure and Macroscopic Properties. Springer, New York, 2002.

[4] D. Westhoff, J. J. Van Franeker, T. Brereton, D. P. Kroese, R. A. J. Janssen, and V. Schmidt. Stochastic modeling and predictive simulations for the microstructure of organic semiconductor films processed with different spin coating velocities. Modelling and Simulation in Materials Science and Engineering, 23(4):045003, 2015.

[5] O. Stenzel, M. Neumann, O. M. Pecho, L. Holzer, and V. Schmidt. Big data for microstructureproperty relationships: A case study of predicting effective conductivities. AIChE Journal, 63(9):4224-4232, 2017. 
[6] N. Wagner, M. Schulze, and E. Gülzow. Long term investigations of silver cathodes for alkaline fuel cells. Journal of Power Sources, 127(1-2):264-272, 2004.

[7] I. Moussallem, S. Pinnow, N. Wagner, and T. Turek. Development of high-performance silverbased gas-diffusion electrodes for chlor-alkali electrolysis with oxygen depolarized cathodes. Chemical Engineering and Processing: Process Intensification, 52:125-131, 2012.

[8] J. R. Wilson, J. S. Cronin, S. A. Barnett, and S. J. Harris. Measurement of three-dimensional microstructure in a $\mathrm{LiCoO}_{2}$ positive electrode. Journal of Power Sources, 196(7):3443-3447, 2011.

[9] L. Zielke, T. Hutzenlaub, D. R. Wheeler, C.-W. Chao, I. Manke, A. Hilger, N. Paust, R. Zengerle, and S. Thiele. Three-phase multiscale modeling of a $\mathrm{LiCoO}_{2}$ cathode: Combining the advantages of FIB-SEM imaging and X-Ray tomography. Advanced Energy Materials, $5(5), 2015$.

[10] J. R. Wilson, W. Kobsiriphat, R. Mendoza, H.-Y. Chen, J. M. Hiller, D. J. Miller, K. Thornton, P. W. Voorhees, S. B. Adler, and S. A. Barnett. Three-dimensional reconstruction of a solidoxide fuel-cell anode. Nature Materials, 5(7):541-544, 2006.

[11] B. S. Prakash, S. S. Kumar, and S. T. Aruna. Properties and development of Ni/YSZ as an anode material in solid oxide fuel cell: A review. Renewable and Sustainable Energy Reviews, 36:149-179, 2014.

[12] M. Neumann, J. Staněk, O. M. Pecho, L. Holzer, V. Beneš, and V. Schmidt. Stochastic 3D modeling of complex three-phase microstructures in SOFC-electrodes with completely connected phases. Computational Materials Science, 118:353-364, 2016.

[13] B. Kenney, M. Valdmanis, C. Baker, J. G. Pharoah, and K. Karan. Computation of tpb length, surface area and pore size from numerical reconstruction of composite solid oxide fuel cell electrodes. Journal of Power Sources, 189(2):1051-1059, 2009.

[14] Q. Cai, C. S. Adjiman, and N. P. Brandon. Modelling the 3D microstructure and performance of solid oxide fuel cell electrodes: Computational parameters. Electrochimica Acta, 56(16):5804-5814, 2011.

[15] H. Ravash, J. Vleugels, and N. Moelans. Three-dimensional phase-field simulation of microstructural evolution in three-phase materials with different interfacial energies and different diffusivities. Journal of Materials Science, 52(24):13852-13867, 2017.

[16] B. Abdallah, F. Willot, and D. Jeulin. Morphological modelling of three-phase microstructures of anode layers using SEM images. Journal of Microscopy, 263(1):51-63, 2016.

[17] H. Moussaoui, J. Laurencin, Y. Gavet, G. Delette, M. Hubert, P. Cloetens, T. Le Bihan, and J. Debayle. Stochastic geometrical modeling of solid oxide cells electrodes validated on 3D reconstructions. Computational Materials Science, 143:262-276, 2018.

[18] M. Neumann, B. Abdallah, L. Holzer, F. Willot, and V. Schmidt. Stochastic 3D modeling of three-phase microstructures for the prediction of transport properties in solid oxide fuel cells. Submitted, 2018+. 
[19] C. Lantuéjoul. Geostatistical Simulation: Models and Algorithms. Springer, Berlin, 2013.

[20] M. Armstrong, A. Galli, H. Beucher, G. Loc'h, D. Renard, B. Doligez, R. Eschard, and F. Geffroy. Plurigaussian Simulations in Geosciences. Springer, New York, 2011.

[21] R. J. Adler. The Geometry of Random Fields. J. Wiley \& Sons, Chichester, 1981.

5 [22] G. Le Loc'h and A. Galli. Truncated plurigaussian method: theoretical and practical points of view. In E. Y. Baafi and N. A. Schofield, editors, Geostatistics Wollongong '96, volume 1, pages 211-222. Kluwer, Dordrecht, 1997.

[23] I. Moussallem, J. Jörissen, U. Kunz, S. Pinnow, and T. Turek. Chlor-alkali electrolysis with oxygen depolarized cathodes: history, present status and future prospects. Journal of Applied Electrochemistry, 38(9):1177-1194, 2008.

[24] J. Kintrup, M. Millaruelo, V. Trieu, A. Bulan, and E. S. Mojica. Gas diffusion electrodes for efficient manufacturing of chlorine and other chemicals. The Electrochemical Society Interface, $26(2): 73-76,2017$.

[25] P. Schmittinger (ed.). Chlorine: Principles \& Industrial Practice. J. Wiley \& Sons, Chichester, 2008.

[26] T. F. O'Brien, T. V. Bommaraju, and F. Hine. Handbook of Chlor-Alkali Technology, volume 1. Springer, New York, 2007.

[27] S. N. Chiu, D. Stoyan, W. S. Kendall, and J. Mecke. Stochastic Geometry and its Applications. J. Wiley \& Sons, Chichester, 3rd edition, 2013.

[28] F. Ballani, Z. Kabluchko, and M. Schlather. Random marked sets. Advances in Applied Probability, 44(3):603-616, 2012.

[29] J. Ohser and K. Schladitz. 3D Images of Materials Structures: Processing and Analysis. J. Wiley \& Sons, Weinheim, 2009.

[30] L. F. Shampine. Vectorized adaptive quadrature in MATLAB. Journal of Computational and Applied Mathematics, 211(2):131-140, 2008.

[31] L. F. Shampine. MATLAB program for quadrature in 2D. Applied Mathematics and Computation, 202(1):266-274, 2008.

[32] MATLAB 2015b, The MathWorks. www.matlab.com, 2015.

[33] Z. Drezner and G. O. Wesolowsky. On the computation of the bivariate normal integral. Journal of Statistical Computation and Simulation, 35(1-2):101-107, 1990.

[34] A. Genz. Numerical computation of rectangular bivariate and trivariate normal and t probabilities. Statistics and Computing, 14(3):251-260, 2004.

[35] M. Salzer, A. Spettl, O. Stenzel, J.-H. Smått, M. Lindén, I. Manke, and V. Schmidt. A twostage approach to the segmentation of FIB-SEM images of highly porous materials. Materials Characterization, 69:115-126, 2012. 
[36] M. Salzer, S. Thiele, R. Zengerle, and V. Schmidt. On the importance of FIB-SEM specific segmentation algorithms for porous media. Materials Characterization, 95:36-43, 2014.

[37] A. Buades, B. Coll, and J.-M. Morel. A non-local algorithm for image denoising. In IEEE Computer Society Conference on Computer Vision and Pattern Recognition (CVPR 05), volume 2, pages 60-65. IEEE, 2005.

[38] J. Schindelin, I. Arganda-Carreras, E. Frise, V. Kaynig, M. Longair, T. Pietzsch, S. Preibisch, C. Rueden, S. Saalfeld, B. Schmid, J.-Y. Tinevez, D. W. White, V. Hartenstein, K. Eliceiri, P. Tomancak, and A. Cardona. Fiji: an open-source platform for biological-image analysis. Nature Methods, 9(7):676, 2012.

[39] N. Otsu. A threshold selection method from gray-level histograms. IEEE Transactions on Systems, Man, and Cybernetics, 9(1):62-66, 1979.

[40] B. Münch and L. Holzer. Contradicting geometrical concepts in pore size analysis attained with electron microscopy and mercury intrusion. Journal of the American Ceramic Society, 91:4059-4067, 2008.

[41] P. Soille. Morphological Image Analysis: Principles and Applications. Springer, New York, 2003.

[42] G. Matheron. Random Sets and Integral Geometry. J. Wiley \& Sons, New York, 1975.

[43] M. B. Clennell. Tortuosity: a guide through the maze. Geological Society, London, Special Publications, 122:299-344, 1997.

[44] L. Holzer, B. Iwanschitz, T. Hocker, L. Keller, O. M. Pecho, G. Sartoris, P. Gasser, and B. Münch. Redox cycling of Ni-YSZ anodes for solid oxide fuel cells: Influence of tortuosity, constriction and percolation factors on the effective transport properties. Journal of Power Sources, 242:179-194, 2013.

[45] M. Neumann, C. Hirsch, J. Staněk, V. Beneš, and V. Schmidt. Estimation of geodesic tortuosity and constrictivity in stationary random closed sets. Scandinavian Journal of Statistics, Under Revision, 2018.

[46] O. Stenzel, O. M. Pecho, L. Holzer, M. Neumann, and V. Schmidt. Predicting effective conductivities based on geometric microstructure characteristics. AIChE Journal, 62:1834-1843, 2016.

[47] L. Holzer, O. Pecho, J. Schumacher, P. Marmet, O. Stenzel, F. N. Büchi, A. Lamibrac, and B. Münch. Microstructure-property relationships in a gas diffusion layer (GDL) for polymer electrolyte fuel cells, part I: Effect of compression and anisotropy of dry GDL. Electrochimica Acta, 227:419-434, 2017. 